\title{
Changes in symphysis pubis width during labor
}

\author{
Shahnoza Rustamova, Mladen Predanic, \\ Melanie Sumersille and Wayne R. Cohen* \\ Departments of Obstetrics and Gynecology, Jamaica \\ Hospital Medical Center and the Weill Cornell Medical \\ College, New York, USA
}

\begin{abstract}
We studied changes in the width of the symphysis pubis in 32 women examined serially by ultrasound during labor. Measurements were made at the superior border of the symphysis and at its narrowest breadth in the latent phase, the active phase, and the second stage of labor. There was a significant increase in the width of the symphysis between the first and second stages of labor at both measured levels. Widening was observed in $94 \%$ at the superior symphyseal breadth and in $59 \%$ at the narrowest. Of those cases in which the width of the symphysis increased, there was a large spectrum of change, ranging from 9 to $98 \%$ of the original width at the narrowest measurement site and from 2 to $139 \%$ at the superior breadth. There was a strong inverse correlation between maternal age and the degree of symphyseal widening in nulliparas, but not in multiparas. We conclude that labor is associated with a substantial widening of the symphysis pubis in most, but not all women.
\end{abstract}

Keywords: Labor; pelvic joints; symphysis pubis.

\section{Introduction}

Evaluation of the structure of the pelvis has, in various forms, always been used to guide obstetric practice. The bony architecture of the pelvis is in fact a crucial element in determining the mechanism of labor and the likelihood of safe vaginal delivery. A clear understanding of the structure and dynamic anatomy of the pelvis is therefore important if the most informed decisions about labor and delivery are to be made.

Considerable rigidity and strength of the pelvic articulations are necessary for normal locomotion and sup-

\footnotetext{
${ }^{*}$ Corresponding author:

Wayne R. Cohen, MD

Jacobi Medical Center

1400 Pelham Parkway South

Room 1 N30

Bronx

New York 10461

USA

Tel.: + 1-718-918-4567

Fax: +1-718-918-8765

E-mail: wayne.cohen@nbhn.net
}

port of the axial skeleton, and under most circumstances there is minimal movement in the sacroiliac joints or the symphysis pubis. There is considerable evidence, nevertheless, that some relaxation of the pelvic joints occurs during pregnancy $[1,2,10,15]$. With specific regard to the symphysis pubis, several studies have shown that its width increases in most women during pregnancy [1, 2, $5,16,17]$, although this finding has not been universal [11].

While the symphysis pubis probably widens during pregnancy, it is less clear whether and to what extent the symphyseal gap expands during labor, as a possible adaptation to foster passage of the fetus. Borell and Fernström [6, 7] concluded, based on an extensive series of intrapartum X-ray studies, that widening of the symphysis was not a common or important part of the expansion of pelvic diameters observed during labor, and other investigators found minimal intrapartum change in the symphyseal breadth $[4,17]$. Some studies are, however, consistent with substantial widening of the symphysis during labor [8, 10].

To help clarify this issue, we serially studied a sample of women during labor to assess the dynamic parturitional features of the symphysis pubis. We used ultrasound to measure the span of their symphysis at two anatomic levels three times during the course of labor.

\section{Materials and methods}

Authorization for this observational study was obtained from the Jamaica Hospital Medical Center's Institutional Review Board. A convenience sample of 32 subjects with singleton pregnancies at gestational weeks 37-42 was recruited in early labor. All had cephalic presentations. We excluded women with a prior cesarean delivery, a history of symphysis pubis separation, or current symphyseal pain. After obtaining informed consent, a series of three measurements of the symphysis pubis width was obtained between uterine contractions in each subject: 1) in the latent phase (cervical dilatation 1-4 cm); 2) in the active phase (cervical dilatation 5-9 cm); and 3) in the second stage, within one hour of complete cervical dilatation. All patients were actively bearing-down during second stage contractions. The phase of labor in which the measurements were made was determined according to Friedman's criteria [9].

To insonate the symphysis pubis, we used a GE LOGIQ 200 PRO ultrasound unit, with a $3.5 \mathrm{MHz} 3 \mathrm{Cb}$ transabdominal transducer (General Electric Healthcare, Waukesha, WI, USA). With the subject supine and her hips flexed about $15^{\circ}$, the probe was placed at the upper border of the symphysis and angled caudad. In this manner a clear view of the upper portion of the symphyseal cleft and its bordering pubic bones was obtained in all subjects. 
Two measurements were acquired at each examination using the electronic calipers of the machine. The first was the distance between the medial margins of the superior pubic rami at the upper boundary of the symphysis, i.e., immediately inferior to the inferred location of the superior pubic ligament (superior symphyseal breadth); the second was made at the narrowest visualized portion of the symphyseal gap (narrowest symphyseal breadth) (Figure 1). All measurements were made in real-time by a single author; later, a second author blinded to the subject's identity or stage of labor verified that the images were of good quality and that the calipers had been placed uniformly in each case.

Pertinent information about the subjects was abstracted from their electronic medical records (E\&C Medical Intelligence, Inc., New York, NY, USA). All data were entered into a statistical data base (JMP Statistical Software 7.0; SAS Institute, Cary, NC, USA) for analysis. Differences among the measurements obtained at the three intervals during labor were sought using one-way repeated measures analysis of variance (ANOVA). The Student-Newman-Keuls test was employed to identify differences between individual groups in the ANOVA. Pearson correlation coefficients were calculated to assess the relationship between the change in the symphyseal width and other continuous
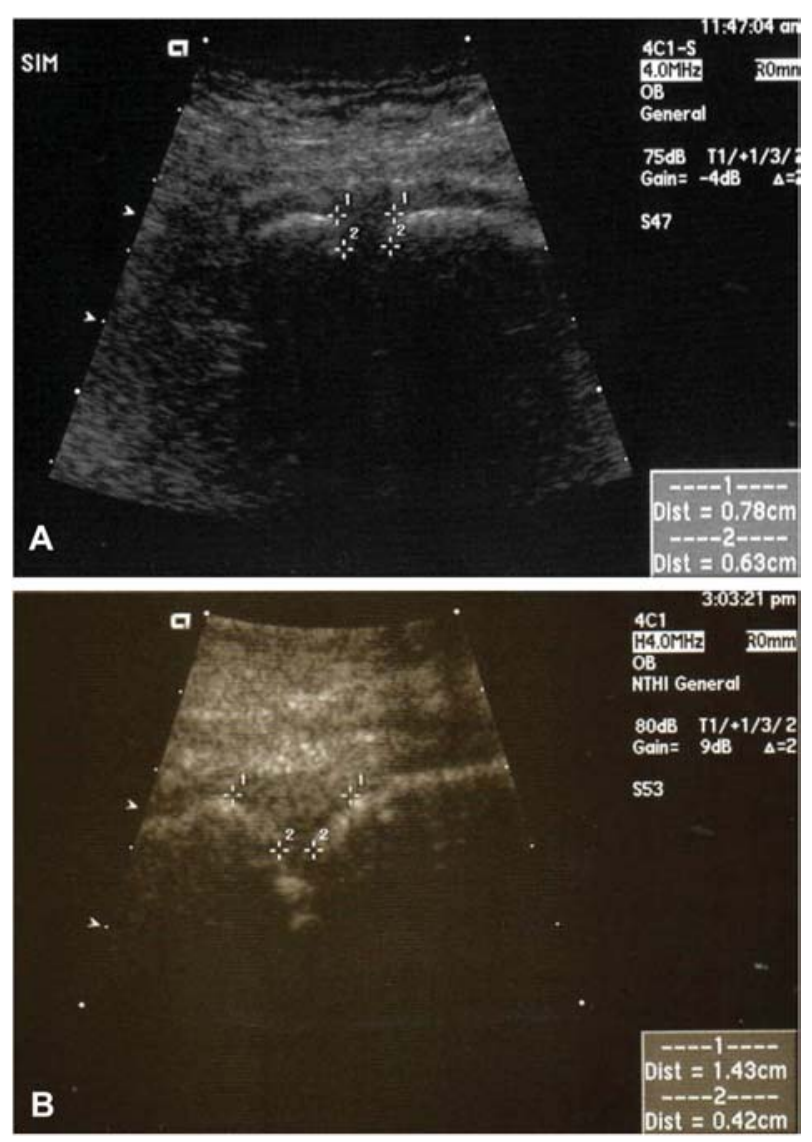

Figure 1 Ultrasonic view of the superior portion of the symphysis pubis demonstrating the two measurement levels used in the study in a "T-shaped" (A) and a "Y-shaped" (B) superior symphyseal conformation. variables, including birth weight, maternal body mass index (BMI), age, and parity. Data are presented as mean \pm standard deviation, unless otherwise specified.

\section{Results}

Characteristics of the study sample are presented in Table 1. Age of subjects ranged from 14 to 44 years, and they were predominantly multiparous $(62 \%)$. Three had oxytocin for labor augmentation, and three were delivered by cesarean during the second stage because of concerns about abnormal fetal heart rate patterns. The remaining 29 patients delivered spontaneously. Birth weights ranged from 2870 to $4200 \mathrm{~g}$.

The difference in the symphyseal gap among the three measurement groups was highly significant for both the superior and the narrowest symphyseal breadth (SB) measurements (Table 2). Multiple comparison testing showed that this difference was accounted for by the change between measurements done in the latent phase and active phase when compared to the second stage measurements. The difference in symphyseal breadth between the latent and active phases of the first stage of labor was not significant. There was no correlation between the degree of expansion of the symphysis in the active phase and the degree of attained cervical dilatation $(P=0.344)$.

Although the overall changes were significantly different, the symphyseal cleft did not expand in all patients. Widening was observed in $94 \%$ at the superior SB measurement and in 59\% at the narrowest SB. Among cases in which symphyseal gap distension was noted, the median change in the SB dimension was $28 \%$ of the original superior diameter and $31 \%$ of the narrowest diameter. In some subjects the observed SB change was minimal, while in others the original gap more than doubled in width (Table 3). The changes in the SB measurements during labor did not differ by parity, and the latent phase SB measurements were not significantly different between nulliparas and multiparas.

Table 1 Characteristics of the sample $(n=32)$.

\begin{tabular}{ll}
\hline Age (years) & $25.7 \pm 8$ \\
Parity (\%, multiparas) & $62 \%$ \\
Body mass index $\left(\mathrm{kg} / \mathrm{m}^{2}\right)$ & $29.7 \pm 4.4$ \\
Race/ethnicity $\mathrm{n}(\%)$ & \\
$\quad$ Black & $11(34 \%)$ \\
Hispanic & $16(50 \%)$ \\
$\quad$ South Asian & $5(15 \%)$ \\
Delivery mode & \\
$\quad$ Spontaneous vaginal $\mathrm{n}(\%)$ & $29(91 \%)$ \\
Cesarean n (\%) & $3(9 \%)$ \\
Oxytocin use n (\%) & $3(16 \%)$ \\
Birth weight (g) & $3401 \pm 367$ \\
Gestational age (weeks) & $39.3 \pm 1.7$ \\
\hline
\end{tabular}


Table 2 Symphyseal breadth (cm).

\begin{tabular}{lllll}
\hline Symphyseal breadth & Latent phase & Active phase & Second stage & P-value \\
\hline Narrowest & $0.99 \pm 0.47$ & $1.05 \pm 0.47$ & $1.21 \pm 0.59$ & 0.007 \\
Superior & $1.60 \pm 0.50$ & $1.66 \pm 0.49$ & $2.07 \pm 0.56$ & 0.0001 \\
\hline
\end{tabular}

Table 3 Change in symphyseal breadth in cases in which it expanded during labor.

\begin{tabular}{lll}
\hline Symphyseal breadth & Change in gap $(\mathrm{cm})^{\star}$ & $\%$ Change (range) \\
\hline Narrowest $(n=19)$ & $0.33(0.08,1.05)$ & $31(9-98)$ \\
Superior $(n=30)$ & $0.46(0.06,1.03)$ & $28(2-139)$ \\
\hline
\end{tabular}

${ }^{*}$ Median $\left(10^{\text {th }}, 90^{\text {th }}\right.$ percentiles $)$.

The SB diminished between the latent phase and second stage at the narrowest measurement strait in $16 \%$ and at the superior SB in $6 \%$ of subjects. It remained unchanged in $25 \%$ of cases at the narrow dimension and in none at the superior measurement. In those women in whom the symphyseal gap decreased, the median reduction in diameter was $0.3 \mathrm{~cm}$. The largest observed change was an increase of $1.13 \mathrm{~cm}$ at the superior SB and $1.5 \mathrm{~cm}$ at the narrowest SB.

There was not a significant correlation between the change in the symphyseal breadth according to birth weight, parity or maternal BMI. When we examined the relationship between the change in the symphyseal width between the latent phase and the second stage according to maternal age and parity, we found a strong negative correlation in nulliparas $(r=-0.85, P=0.0004)$ but not in multiparas $(r=0.22, P=0.345)$.

\section{Discussion}

The width of the symphysis increased significantly overall in our sample during the second stage of labor. While the mean SB was greater in the active phase than in the latent phase of the first stage, this trend was not significant. Our data are consistent with those of other investigators who suggested that the symphysis may widen during labor $[8,10,16]$. We also have confirmed that changes in the symphysis occur to a varying degree in most women, but are not universal.

Most previous efforts to assess changes in the bony pelvis during labor were done with radiography. Thorp and Fray [16], for example, showed widening of the symphyseal gap in $44 \%$ of cases in the first stage of labor compared to an evaluation done in the third trimester prior to labor. Brehm and Weirauk [8] found an increase in the width of the symphysis in $54 \%$ of patients $\mathrm{X}$-rayed before and after delivery. However, Young [17] found no appreciable effect of labor on the symphyseal breadth, and Ohlsén [12] identified a change of only about $1 \mathrm{~mm}$ in a few patients. Differences among results of various studies might relate to the difficulty of correcting for magnification in radiographic images, which is influenced by the distance of the measured object from the film. It is also difficult to compare results of studies because measurements were not always taken with subjects in a standardized position. Different postures may alter object-film distances, and change pelvic dimensions. We used ultrasound because it allowed uniformity in positioning of the subjects, in addition to being riskless and causing no discomfort.

Ultrasound was shown by Bjõrkland et al. in an anatomic model to have precision equivalent to that of radiography in measuring the symphysis pubis [3]. They used ultrasound to study the pubic symphysis during labor at the time of engagement and then in the second stage [4]. They found a very modest increase (averaging $<1 \mathrm{~mm}$ ) in the symphyseal breadth in most subjects, and no change or a narrowing in $9 \%$. Their findings may have been compromised by the fact that subjects were not always studied in the same posture at both time points in labor. Moreover, some had experienced pelvic pain during the pregnancy studied, suggesting that they may have had symphyseal pathology.

Our subjects were evaluated in the supine position with mild hip flexion for all three measurements. None complained of pubic pain during pregnancy, labor or the postpartum period. We did not define the subjects' pelvic types in our study, and were therefore unable to determine whether pelvic architecture influenced changes in the symphyseal breadth during parturition.

Each delivery causes some alteration of the symphyseal bone and fibrocartilage. In addition to inducing degenerative arthritic changes, each pregnancy might render the pelvic joints more lax [13]. In addition, age causes changes in the symphysis, regardless of parity [13]. This age-associated loss of elasticity and progressive fibrosis in the joint might result in reduced ability of the pelvis in reproductively older nulliparas to mold optimally as the fetus attempts its odyssey down the birth canal. Our data showing an inverse relation between age and the parturitional widening of the symphyseal gap in nulliparas, but not multiparas, would be consistent with this notion. The multipara may have changes in the supporting ligaments that render the symphysis more distensible regardless of age.

Our measurements are generally consistent with symphyseal gaps noted by several other investigators using 
radiographs or ultrasound $[1,8,10,11,14]$. Measurement can be difficult to standardize because of the frequently observed pubic bone surface irregularities and the fact that the bony margins of the symphysis are not always parallel. Also, the profile of the superior portion of the symphysis can take two general shapes (Figure 1b). In some women the medial edges of the pubic bones descend caudad in an arc, giving the upper portion of the symphysis a Y-shaped appearance; in others the borders of the symphysis are parallel throughout, conferring a T-shape to the superior SB. To address the potential difficulty this might introduce in comparing dimensions between subjects, we used two measurements: the distance between the superior pubic rami, and the narrowest portion of the symphyseal gap visualized in our scans. The minimal SB was not influenced by the configuration of the upper portion of the symphysis. Both measurements changed in the same direction in most subjects, regardless of the symphyseal joint shape. The fact that the superior SB enlarged in more subjects than the narrowest SB suggests that symphyseal widening may not occur symmetrically.

In summary, we have demonstrated that the pubic symphysis widens during labor in most women, and that these changes can be readily assessed by ultrasound. The dynamic properties of the joint are presumably a physiologic adaptation to ease the passage of the fetus through the birth canal. It seems most likely that the observed changes are a passive response to the forces created by the descending fetus, acting on the joint and its surrounding ligaments, the compliance of which has been increased by hormonal changes of pregnancy. We speculate that the extensibility of the pelvic joints during the second stage of labor may be an important factor in determining the probability of a safe vaginal delivery.

\section{References}

[1] Abramson D, Roberts SM, Wilson PD. Relaxation of the pelvic joints in pregnancy. Surg Gynecol Obstet. 1934; 58:595-613.

[2] Bahlmann F, Merz E, Macchiella D, Weber G. Sonographische darstellung des symphysenspaltes zur beurteilung eines symphysenschadens in der schwangerschaft und post partum. Z Geburtshilfe Perinatol. 1993;197:27-30.

[3] Bjõrklund K, Bergström S, Lindgren PG, Ulmsten U. Ultrasonographic measurement of the symphysis pubis: $A$ potential method of studying symphyseolysis in pregnancy. Gynecol Obstet Invest. 1996;42:151-3.

[4] Bjorklund K, Lindgren PG, Bergstrom S, Ulmsten U. Sonographic assessment of symphyseal joint distention intra partum. Acta Obstet Gynecol Scand. 1997;76:227-32.

[5] Bjõrklund K, Nordstrom ML, Bergstrom S. Sonographic assessment of symphyseal joint distention during pregnancy and post partum with special reference to pelvic pain. Acta Obstet Gynecol Scand. 1999;78:125-30.

[6] Borell U, Fernström I. The movements at the sacro-iliac joints and their importance to changes in the pelvic dimensions during parturition. Acta Obstet Gynecol Scand. 1957;36:42-57.

[7] Borell U, Fernström I. Pelvimetric method for the assessment of pelvic 'mouldability'. Acta Radiol. 1957;47:365-9.

[8] Brehm W, Weirauk HV. Separation of the symphysis pubis during labor. Am J Obstet Gynecol. 1928;15:187-91.

[9] Friedman EA. Labor: Clinical Evaluation and Management, second edition. Appleton-Century-Crofts, New York, 1978.

[10] Garagiola DM, Tarver RD, Gibson L, Rogers RE, Wass JL. Anatomic changes in the pelvis after uncomplicated vaginal delivery: a CT study on 14 women. Am J Rhinol. 1989; 153:1239-41.

[11] Heyman J, Lundqvist $A$. The symphysis pubis in pregnancy and parturition. Acta Obstet Gynecol Scand. 1932;12: 191-226.

[12] Ohlsén $\mathrm{H}$. Moulding of the pelvis during labour. Acta Radiol Diagn. 1973;14:417-34

[13] Putschar WG. The structure of the human symphysis pubis with special consideration of parturition and its sequelae. Am J Phys Anthropol. 1976;45:589-94.

[14] Scriven MW, Jones DA, McKnight L. The importance of pubic pain following childbirth: a clinical and ultrasonographic study of diastasis of the pubic symphysis. J R Soc Med. 1995;88:28-30.

[15] Thoms $\mathrm{H}$. Relaxation of the symphysis pubis in pregnancy. J Am Med Assoc. 1936;106:1364-6.

[16] Thorp D, Fray W. The pelvic joints during pregnancy and labor. J Am Med Assoc. 1938;11:1162-6.

[17] Young J. Relaxation of the pelvic joints in pregnancy. J Obstet Gynaec Br Emp. 1940;47:493-524.

The authors stated that there are no conflicts of interest regarding the publication of this article.

Received June 19, 2008. Revised October 27, 2008. Accepted November 7, 2008. Previously published online March 17, 2009. 